\title{
The Physics and the Philosophy of Time Reversal in Standard Quantum Mechanics
}

\author{
Cristian López \\ University of Lausanne, Department of Philosophy \\ University of Buenos Aires, Institute of Philosophy \\ CH-1015 Lausanne, Switzerland \\ lopez.cristian1987@gmail.com
}

(Draft August 01, 2020)

\begin{abstract}
A widespread view in physics holds that the implementation of time reversal in standard quantum mechanics must be given by an anti-unitary operator. In foundations and philosophy of physics, however, there has been some discussion about the conceptual grounds of this orthodoxy, largely relying on either its obviousness or its mathematicalphysical virtues. My aim in this paper is to substantively change the traditional structure of the debate by highlighting the philosophical commitments underlying the orthodoxy. I argue the persuasive force of the orthodoxy greatly depends on a relationalist metaphysics of time and a by-stipulation view of time-reversal invariance. Only with such philosophical background can the orthodoxy of time reversal in standard quantum mechanics succeed and be properly justified.
\end{abstract}

Keywords: quantum mechanics, time reversal, anti-unitarity, relationalism, symmetry.

\section{Introduction}

What grounds the claim that a quantum physical system has been genuinely time reversed? Answers to this question fall in either of two sides. On the one hand, the overarching attitude (the orthodoxy, henceforth) points out that in order to reverse the dynamical evolution of a quantum system, an anti-unitary time-reversal operator must be given. On the other hand, some philosophers have lately argued that such orthodoxy might be challenged (Albert 2000, Callender 2000, Lopez 2019), which has paved the way for non-standard representations of time reversal in quantum mechanics (QM, henceforth), in general, in terms of a unitary implementation. 
In one way or another, a thorough response to the opening question amounts to spelling the notion of time reversal out. This has led to a reinforcement of the orthodoxy by providing a precise mathematical tailoring of the implementation of time reversal as well as a more attentive philosophical refinement of its foundations (see, for instance, Sachs 1987, Earman 2002, Roberts 2017, 2018). The orthodoxy has generally been defended as the only view that is philosophically and physically viable, centering its defense in showing that a non-standard implementation of time reversal (i.e. that provided by a unitary time-reversal operator) fails to deliver a workable mathematical transformation as well as a conceptually defensible notion of time reversal. So, the dispute has been mostly set in terms of a unitary versus an anti-unitary implementation of time reversal in QM, where the orthodoxy champions the latter, while non-standard views (or 'heretic' views), the former.

Yet, I believe that the dispute between a unitary versus an anti-unitary implementation of time reversal is just the top of the iceberg in a series of philosophical and physical decisions that have to be made in order to conceptualize the idea of time reversal and to formally represent it. The bone of contention is not whether an anti-unitary implementation more genuinely represents time reversal simpliciter, but which concept of time reversal it intends to model mathematically, which are its philosophical assumptions, and whether they are defensible. To state it differently, much of the persuasive force of the orthodoxy crucially depends upon a series of philosophical and physical decisions within which the anti-unitary implementation of time reversal naturally makes sense. To thoroughly comprehend the nature of time reversal in QM is to bring to light such decisions and assumptions.

The aim of this paper is to substantively change the traditional structure of the debate by bringing to the forefront the philosophical commitments underlying the orthodoxy. My approach to the debate seeks to identify, and follow the trail of, the series of decisions and assumptions that make the orthodox notion of time reversal so defensible. This would not only strengthen the legitimacy of the orthodoxy but would also provide a more accurate picture of how complex the notion of time reversal is and the many unnoticed substantial philosophical assumptions that underpin it. To begin, I will distinguish three steps in building up the notion of time reversal, both conceptually and formally.

1. The mathematical tailoring, whereby the anti-unitary implementation comes out as a fair modeling of time reversing a quantum system.

2. The physical justification, which defends the anti-unitary implementation by stipulating the physical requirements for time reversing a quantum system. This step sets a physics-based concept of time reversal greatly grounded in the idea of 'backtracking'.

3. The philosophical background, which lays the philosophical groundwork for such a physics-based concept of time reversal. This step justifies why the concept of time reversal must be understood as the orthodoxy says it must be understood. 
I will next show that there are two major arguments that physically support the orthodox understanding of time reversal in terms of backtracking, namely, (a) the two-time-evolution argument (or Wigner's general criterion for time reversal), and (b) the Hamiltonian's spectrum argument. But, the central claim of this article comes when the philosophical background against which the physical justification makes sense is brought to light. I will argue that the philosophical background primarily consists in responding the two following questions:

- What do we mean by time?

- What heuristic and epistemic status do we suppose that symmetries have in physics?

Neither of these questions admits a univocal answer. The first question opens a metaphysical dimension in our understanding of time reversal. At this point, I will argue that a relationalist metaphysics of time underlies the orthodoxy, which motivates a functional reductionist approach to time reversal, whereby time reversal ought to be functionally reduced to motion reversal. The second question concerns whether time-reversal symmetry is to be conceived either as a by-stipulation or a by-discovery symmetry. I will argue that both the physical justification and the mathematical tailoring of the orthodoxy sensibly depend on taking timereversal invariance as a by-stipulation symmetry.

The upshot is that the persuasive force of the orthodoxy crucially relies upon this philosophical background, which, naturally, could be challenged. This suggests that the orthodoxy is not the only physical and philosophical viable view in QM, but it is the most natural one given a well-articulated philosophical background. This claim might be welcomed among those holding a heretic attitude to time reversal and might in the same proportion cause some discomfort among those defending the orthodoxy. It must be made clear nonetheless that it is not my intention to directly favor (or disfavor) one of the two, but to expose the various philosophical and physical assumptions that underly the orthodoxy. The general aim is to draw philosophers' and physicists' attention to those philosophical and physical assumptions that support it, instead of showing it wrongheaded. Whether or not those that have challenged the orthodoxy might find in this paper some fertile terrain to develop their heresies further is something I will not discuss here.

The structure of the article is as follows. In Section 2, I will begin by briefly introducing the mathematical tailoring of the orthodoxy of time reversal in QM. I will also show here how a non-standard account can come up. In Section 3, I will expose in detail the two major arguments that physically supports the orthodoxy. In Section 4, I will provide the main arguments of the paper by offering the philosophical background the orthodoxy is framed within. It chiefly consists of two pillars -a metaphysical pillar (Section 4.1) and a heuristicepistemic one (Section 4.2.). Finally, concluding remarks. 


\section{The Mathematical Tailoring of the Orthodoxy}

In the Hamiltonian formulation of classical mechanics, the main features of the time-reversal transformation stem from an analysis of the physics of the simplest cases. So, the starting point is typically a particle moving on a line in a conservative field force. The state of the particle is given by two variables: the generalized coordinates $q_{i}$ and the conjugate momenta $p_{i}$. So, a trajectory in the phase space will be described through a set of functions $q_{i}(t), p_{i}(t)$, which is given by the Hamiltonian of the free particle:

$$
H=\frac{p^{2}}{2 m}+V(x)
$$

As $V(x)$ is constant, it plays no role and we can disregard it. In their most general expression, the Hamilton's equations follow from a system's Hamiltonian as function of the $q_{i} \mathrm{~s}$ and $p_{i} \mathrm{~s}$

$$
\dot{p}_{i}=-\frac{\partial H}{\partial q_{i}} ; \quad \dot{q}_{i}=\frac{\partial H}{\partial p_{i}}
$$

So, in which way can the time-reversal transformation be implemented? The answer mostly depends on what time reversing a classical system means, conceptually. Even though there would be much to say here, the most common answer, and one that is quite easy to grasp, is that of a film played backward. So, by time reversing a classical system we mean to generate a transformation that retraces the trajectory of a system This is the guiding concept that we want to formally implement. Such an implementation is what I will call the 'mathematical tailoring', that is, the process whereby such a concept is formally modeled within a theory. To put it more accurately, it is the process whereby a mathematical representation is given with the right sort of properties to capture what we conceptually mean by 'time reversing' within a theory. Canonically, the mathematical tailoring of time reversal in Hamiltonian classical mechanics involves a transformation $T$ such that reparametrizes the time coordinate, changes the sign of the $p_{i} \mathrm{~s}$, and leaves $q_{i}$ s unchanged.

$$
T: t \rightarrow-t ; p_{i} \rightarrow-p_{i} ; q_{i} \rightarrow q_{i}
$$

This transformation is directly related to a symmetry property of the Hamiltonian

$$
H\left(q_{i}, p_{i}\right)=H\left(q_{i},-p_{i}\right)
$$

If a system's Hamiltonian satisfies (4), then the equations of motion are invariant under $T$.

When we move to QM, the mathematical tailoring of time reversal "should", at least in principle, follow a similar procedure. We do not know yet what is exactly the quantum concept of time reversal we want to formally represent, but we can nonetheless move forward and take the classical case as a guide. And here is where the orthodoxy starts off. To begin, it warns us against imitating too closely the classical implementation (see for instance Gibson 
and Pollard 1976, or Ballentine 1998). What this chiefly means is that we should not just relocate what we learnt about time reversal in Hamiltonian classical mechanics and apply it directly to QM. In some sense, the mathematical tailoring has to be worked out all over again. As mentioned previously, this amounts to specifying the sort of properties that the implementation of time reversal should involve. Technically, this has been discussed in terms of whether the time-reversal operator is unitary or anti-unitary.

Consider the Schrödinger equation for a free quantum system. What we can take as a lesson from the classical case is that time reversal might have something to do with the idea of backtracking a system to the original state. So, we want to figure out the form of a transformation that does precisely that job. Thus, we can begin by defining a general symmetry transformation, say $T$. We do not know yet whether $T$ will finally succeed or not. We do not even know what $T$ actually stands for -It might be either a trivial transformation without any physical meaning, or something more fundamental. This is not really important for the moment, and we can nonetheless move forward by specifying what $T$ formally does to the Schrödinger equation. So, as a first condition, let us stipulate that $T$ turns $t$ 's sign around, $T: t \rightarrow-t$. After all, $T$ is meant to be a candidate for time reversal, so it has to somehow change the direction of time.

$$
T H|\psi\rangle=i \hbar \frac{\partial T|\psi\rangle}{\partial T t} \rightarrow H|\psi\rangle=-i \hbar \frac{\partial|\psi\rangle}{\partial t}
$$

We immediately note that $T$ produces a minus sign on the right side of the equation. When physically interpreted, this indicates that something will go fatally wrong with this transformation. For instance, the equation will come out non-invariant under $T$. But we do not want to ruin our equation, so we impose a second condition on $T$-it also takes the complex conjugate $K$.

$$
T H|\psi\rangle=T(-i) \hbar \frac{\partial T|\psi\rangle}{\partial T t}
$$

Which finally yields our $T$-transformed Schrödinger equation.

$$
H\left|\psi^{*}\right\rangle=i \hbar \frac{\partial\left|\psi^{*}\right\rangle}{\partial t}
$$

If $T$ is defined by what it does, the first and second conditions already give us a quite clear picture of the nature of $T$-if a generic quantum state like $|\psi(t)\rangle$ evolves by the Schrödinger equation with $t$ increasing, the $T$-transformed state is given by $\left|\psi^{*}(-t)\right\rangle$, which evolves by the $T$-transformed Schrödinger equation. We can already bet that $T$ will be a viable candidate to implement time reversal. 
It is worth highlighting some further aspects of the $T$-transformation before moving ahead. First, the equation comes out invariant under the application of $T$. One of the reasons for this is that $T$ transforms the Hamiltonian as $T: H \rightarrow H^{*}$, keeping its spectrum invariant under the $T$-transformation. Second, the explicit factor $i$ in the Schrödinger equation plus the requirement that it should leave the modulus of the overlap of two state vectors invariant would, prima facie, explain that $T$ is anti-unitary. Hence, $T$ can be given with the so-called standard form $T=U K$, where $U$ is a unitary operator and $K$ the complex conjugation, $K z K^{-1}=z^{*}$, with respect to a standard representation. Third, we can naturally impose further subsidiary requirements upon $T$. For instance, $T$ can be also demanded to change the sign of momentum, $T \mathbf{P} T^{-1}=-\mathbf{P}$. Finally, it is blatant that the $T$-transformation preserves the transition probabilities as well

$$
\langle T \psi \mid T \varphi\rangle=\langle\psi \mid \varphi\rangle
$$

At this point, the following question may arise: What does $T$ stand for? After all, $T$ in $\mathrm{QM}$ does to a great extent the same job that $T$ did in the classical case -it retraces the system's state to the initial state. So, either unwittingly or wittingly, we have been following the same recipe: we associate the concept of time reversal with the idea of backtracking the state of a system to the original state. Then, the mathematical tailoring in QM just works the form of the operator out that instantiates the right sort of properties to formally implement such a concept. Finally, we stipulate that 'time reversal' is fairly implemented by $T$, binding time reversal (as retracing the quantum state) and $T$ (as its formal implementation) together. And, in so doing, we justify the orthodoxy: time reversal in QM is implemented by an anti-unitary operator, which thereby deserves the name 'time reversal'.

To highlight the distinctiveness of the orthodoxy, let us compare it with a non-standard account of time reversal in QM. It has been generally posed in terms of the mathematical tailoring: time reversal is not fairly given by an anti-unitary operator, but by a unitary one. Formally, it entails that the formal representation of time reversal (let us call it $T^{\prime}$ to distinguish it from $T$ as specified above) does not take the complex conjugation over the state, but it is exhaustively given by the transformation $T^{\prime}: t \rightarrow-t$, which yields

$$
T^{\prime} H|\psi\rangle=i \hbar \frac{\partial T^{\prime}|\psi\rangle}{\partial T^{\prime} t} \rightarrow H|\psi\rangle=-i \hbar \frac{\partial|\psi\rangle}{\partial t}
$$

This is all we have to say about the mathematical tailoring of a non-standard implementation of time reversal: change the sign of $t$ and of all those first-time-derivative magnitudes accordingly. This 'heretic' attitude comes up in the literature in at least two ways:

(a) as an unacceptable way to formally represent time reversal in QM, which has to be dismissed in favor of the orthodoxy (see for instance Gasiorowicz 1966: 27), or 
(b) as a positive defense of a "more genuine" and "broader" way to formally represent time reversal in physics (see Albert 2000, Callender 2000. Costa de Beauregard $1980^{1}$ also defends such a view in quantum field theory).

It is noteworthy that both (a) and (b) center in the mathematical tailoring of the non-standard account of time reversal. According to those supporting (a), a non-standard account fails to offer the right sort of mathematical tailoring for what we should mean by 'time reversal'. Contrarily, those supporting (b) believe that a non-standard account better captures the idea of time reversal, entailing that the binding between the concept of time reversal and its orthodox implementation must be broken. I believe that the orthodoxy is on the right track in this. The reason is that the unitary representation of time reversal would bring about a series of devastating formal and physical consequences that must be at all costs avoided if time reversal means what the orthodoxy wants it to mean. For instance, the unitary representation of time reversal transforms the Hamiltonian's spectrum, which by definition is always invariant. I will come back to this shortly, but it partially explains why the free Schrödinger equation is non-invariant under such implementation of time reversal, and why this can be a problem to consider.

Notwithstanding, it does not mean that the concept of time reversal in terms of backtracking and the firmness of the binding to the anti-unitary implementation be selfexplanatory. Neither are they self-evident. To a great extent, the persuasive force of the orthodoxy is grounded in accepting what it means by 'time reversal' conceptually, that is, that the concept of time reversal is defined as retracing a system's state to the original state. If we accept this core idea, then s; the orthodoxy succeeds not simply because it provides the right sort of mathematical tailoring, but because (once accepted) such core idea it can only be implemented by the mathematical tailoring it proposes. We can view the orthodoxy as a chain of formal, physical and philosophical assumptions that articulate very well to yield a coherent view of time reversal. The mathematical tailoring is just the last link in the chain, which formally adapts, shapes and implements a particular conceptualization of time reversal already at work. Now, what grounds such an understanding of time reversal is to a good extent the focus of what follows.

\section{The Physical Justification of the Orthodoxy}

We know that the orthodox understanding of time reversal is somehow guided by the idea of backtracking. Yet, it is still unclear what this means exactly in physical terms within QM, and why the concept of time reversal ought to be conceived as the orthodoxy claims it ought to be. Though standard textbooks remain largely silent about how these questions could be answered, literature on foundations and philosophy of physics has addressed them in some

\footnotetext{
${ }^{1}$ Beauregard refers to "Racah's operator" as opposed to Wigner's (Costa de Beuregard 1980: 524 and further references therein).
} 
detail. Answers to these questions amounts to justifying the orthodox mathematical tailoring as well as its binding to the idea of 'backtracking'. This justificatory task has probably its origins in the work of Eugene Wigner (1932), and it has been re-elaborated in the last decades (see, for instance, Sachs 1987, Earman 2002 and Roberts 2017).

The justificatory task is not simple, though: in general, the justification proceeds in at least two steps. As I will show shortly, the first step, which is quite more explicit, consists of three arguments aiming to show not only how the idea of time reversal as backtracking should be physically understood, but also that the mathematical tailoring as presented in Section 2 is the only one capable of capturing the most salient features of time reversal as backtracking. This is the physical justification and I will develop it along this section. The second step, which, to the best of my knowledge, has not been sufficiently recognized, consists in the philosophical reasons we might have to conceive time reversal as backtracking. This is what I call the philosophical background and I will address it in Section 4.

Setting aside any classically rooted intuition on time reversal as backtracking, the details of what we physically mean by time reversal must be given within a theoretical framework. There are at least two key arguments ${ }^{2}$ upholding the orthodoxy, namely:

- The 'two time-evolution' argument (or Wigner's general criterion for time reversal)

- The Hamilton's spectrum argument

\subsection{The 'two-time-evolution' argument (or Wigner's general criterion for time- reversal)}

One of the distinctive properties of the time-reversal operator in general is that it is an involution. Mathematically, this means that when time reversal is applied twice, it is equal to the identity. Naturally, this is met by any operator that satisfies $X^{2}=I$, though it is not crystal clear why this is equivalent to the idea of backtracking a system's state to its initial state. The locus classicus of this requirement is the work of Eugene Wigner. In his 1932 book, Group Theory and its Application to the Quantum Mechanics of Atomic Spectra, Wigner imposes a general criterion for time reversal stating that it is a transformation such that, when the following operations are sequentially performed, we obtain the identity. Informally,

\footnotetext{
${ }^{2}$ It can be pointed out that there is a third key argument, to wit, that momentum changes its sign under time reversal. Certainly, this is one of the most salient features of the time reversal implementation in classical mechanics. For the most part though, the reasons why the sign of momentum should change under time reversal in QM are somewhat unclear in the literature. In general, reasons swing back and forth from preserving certain smooth continuity between the classical mechanics and QM, to appealing to its obviousness. Some authors just claim that the transformation follows by definition (Messiah 1966: 667, Sachs 1987, Ballentine 1998: 377-378). A more philosophically refined discussion can be found in Callender (2000) and Roberts (2018). In addition, it can be argued that the transformation of momentum plays a paramount role in the semi-classical limit, mainly in relation to Ehrenfest's theorem. However, this argument, and various versions thereof, does not add anything substantive to the point I want to make in this paper, so I will set it aside.
} 
time displacement by $t \times$ time reversal $\times$ time displacement by $t \times$ time reversal $=\boldsymbol{I}$

And more formally,

$$
T\left[U_{\Delta t_{2}} T\left(U_{\Delta t_{1}} s_{0}\right)\right]=s_{0}
$$

Where $s_{0}$ is the initial state, and $\Delta t_{1}=t_{1}-t_{2}=-t_{2}-t_{1}=\Delta t_{2}$

Wigner's general criterion for a time reversal implementation supposes further structure than a simple involution -the time reversal operator is expected to obtain the original state we started with after producing a time evolution with $t$ increasing and by producing a (formally identical) second time evolution with $t$ decreasing. In other words, the timereversal operator is not only required to give us the same initial state when applied twice, but also to give us the same initial state after temporally evolving the system twice. This is a stronger requirement in so much as the time-reversal transformation is expected to carry out the right sort of transformations to, at least, generate a time evolution with $t$ decreasing. This twofold time evolution is not trivial and defines what a time-reversal transformation is. To put it into a slogan -to be a time-reversal operator is to be an operator that yields the identity after two-time evolutions.

Wigner additionally establishes that any candidate for a time-reversal transformation has to preserve transition probabilities.

$$
|\langle\psi \mid \varphi\rangle|=|\langle T \psi \mid T \varphi\rangle|
$$

There is not much justification in the standard literature on time reversal in QM of why time reversal ought to preserve the transition probabilities. Intuitively, it makes senses since if it were not the case, the second time evolution would no longer be possible. In more specific terms, Wigner postulated that the transition probabilities between two states has an invariant physical sense, so any symmetry should preserve them. Yet, this justification follows from the invariance of time reversal, and not from the time-reversal transformation itself. That is, if a symmetry holds, then transition probabilities must be preserved by the symmetry transformation. But this does not tell us whether a generic time reversal transformation will always preserve transitions probabilities.

How does all this relate to an anti-unitary representation of time reversal in QM? The famous Wigner's theorem states that a symmetry transformation is represented either by a unitary or an anti-unitary operator. As the anti-unitary operator is the only one that satisfactorily meets the general criterion and preserves transition probabilities, the unitary operator is discarded. However, it has been pointed out that Wigner's proof of his theorem was "incomplete" (see Chevalier 2007: 429) and that a correct proof has been given by U. Uhlhorn in 1962, who also generalizes the condition of preserving the probabilities. In a 
nutshell, Uhlhorn's proof replaces the preservation of the transition probabilities by the preservation of orthogonality: any pair of orthogonal states $\langle\psi, \varphi\rangle=0$ remains orthogonal under a symmetry transformation $S,\langle S \psi, S \varphi\rangle=0$. It follows from this that $\langle\psi, \varphi\rangle=$ $\langle S \psi, S \varphi\rangle$ (see Chevalier 2007, Section 5, for a proof of Uhlhorn's theorem). For Chevalier, Uhlhorn generalizes Wigner's proof as he shows that a symmetry transformation preserves the logical structure of a quantum theory, as Uhlhorn himself states in the Introduction of his book.

Following the same logic as before, if time-reversal invariance holds, then any orthogonal pair of states remains orthogonal under time reversal, that is, if $\langle\psi, \varphi\rangle=0$, then $\langle T \psi, T \varphi\rangle=$ 0 . But, once again, the justification hinges upon what we should expect from time-reversal invariance. Bryan Roberts (2017) notes that Uhlhorn's theorem provides a general answer to why transition probabilities must be preserved under time reversal and advances a more convincing answer for why the time-reversal transformation ought to preserve transition probabilities. His argument is quite simple: orthogonality has nothing to do with time reversal as it just relates to "what is possible in an experimental outcome, independently of their time development" (Roberts 2017: 321). So, why should we expect that something completely unrelated to time (as two states being mutually exclusive) be modified by time reversal?

The argument is interesting because it concerns what we should expect from a timereversal transformation independently of whether it yields an invariance or not. It is worth bearing in mind that there is yet an assumption here: such implementation of time reversal is supposed to transform quantum-mechanical states into quantum mechanical states. Even though it is true that orthogonality has nothing to do with time reversal, it does have to do with the notion of state. To put it differently, the time-reversal transformation preserves the notion of quantum-mechanical state ${ }^{3}$.

Let us briefly sum up the "two-evolution-based argument". The formal implementation of time reversal might take two forms: either unitary or anti-unitary. Naturally, we have a wide panoply of transformations that might fit in. So, we need to narrow the possibilities down. Wigner's general criterion is a first step toward such a direction, since it states that whatever the time reversal comes to be, it is a transformation such that it delivers the state we started with after a twofold application and two-time evolutions. As a subsidiary requirement, it is demanded to preserve orthogonality, and thereby, transition probabilities.

\footnotetext{
${ }^{3}$ From a philosophical viewpoint, I think it is not trivial. Even though most symmetries physicists are interested in are required at minimum to transform states into states, we should leave some room for metaphysically possible scenarios in which some transformation fails to transform a state into a physical state. Primitivists with respect to time believe that time is fundamental and defines not only the dynamics of a physical theory, but also is constitutive of its kinematics. Why should they take this requirement as obvious? Does it not discard, from the outset, the notion of time as fundamental? I am not defending this viewpoint here, but I just want to draw the attention towards the non-obviousness of the assumption from a metaphysical viewpoint.
} 


\subsection{The Hamiltonian's spectrum argument}

In Section 2, I mentioned in passing that one of the main virtues of the anti-unitary representation of time reversal is that it leaves the Hamiltonian invariant, $T \boldsymbol{H} T^{-1}=H^{*}$. This requirement is essential for upholding the orthodoxy (e.g. Gasiorowicz 1966: 27, Sachs 1987: 36). As the Hamiltonian represents the energy of the system, its spectrum is supposed to be always positive. But, it happens that the unitary representation of time reversal produces a minus sign on the right side of the equation. Since $T^{\prime}$ is a unitary, linear operator, then $T^{\prime} \mathbf{H} T^{\prime-1}=-H$, entailing that if $|\psi\rangle$ is an eigenstate of the Hamiltonian with energy $E$, then the temporally reversed eigenstate $T^{\prime}|\psi\rangle$ should involve negative energies, $-E$. There is nothing formally wrong with this, but problems pop up as rapidly as we step into its physical meaning: if $T^{\prime}$ fairly represents time reversal in QM, then a Hamiltonian evolving backwards in time would display negative energies.

The big issue with this is that we do not have even a way to make sense of this -by definition, Hamiltonians' spectrum is always positive. Negative energy states are supposed to remain undetected and that their existence would necessarily turn matter unstable. This specifically means that the Hamiltonian must be bounded (from below), that is, $H \geq 0$, and to remain so bounded after any symmetry transformation, if we wish to get a transformed quantum mechanics state. The upshot is that the anti-unitary representation of time reversal is the only one that keeps the Hamiltonian's spectrum invariant ${ }^{4}$.

It is relatively straightforward to connect this argument with Wigner's general criterion. If Hamiltonians must be always bounded from below, the second time translation with $t$ decreasing must be generated by a bounded-from-below Hamiltonian. Otherwise, the time translation would be "physically meaningless" since it involves eigenstates of the Hamiltonian whose spectrum is unbounded from below and there is no way to make sense of it in QM. Putting it drastically, unbounded-from-below Hamiltonians must not even be considered as quantum-mechanics systems. Therefore, the implementation of time reversal is demanded not only to generate a second time evolution with $t$ decreasing, but also to generate a quantum-mechanical meaningful second time evolution, which means that it is generated by $H^{*}$. This just strengthens the necessity of relying on an anti-unitary representation of time reversal. If we define time reversal as we have done thus far, it follows that a unitary representation does not properly capture such a definition.

\footnotetext{
${ }^{4}$ It is worth clarifying that the predicates "positive" or "negative" for the energy spectrum, or "unbounded from below/from above" for Hamiltonians are conventional. So, the argument could not hinge upon which predicate we adopt to describe the system properly. The problem is not exactly whether the Hamiltonian is unbounded from below. The problem is that if we start with a Hamiltonian unbounded from above (but bounded from below) and end up with a Hamiltonian unbounded from below (but bounded from above) after a transformation. A specific Hamiltonian must be bounded (either from above or from below), and the problem would come up if one adopts a transformation that turns a Hamiltonian unbounded from above (bounded from below) into a Hamiltonian unbounded from below (bounded from above).
} 
Roberts (2017) offers more solid grounds for demanding that the Hamiltonian's spectrum ought to remain invariant under time reversal. He begins by claiming that "all known Hamiltonians describing realistic quantum systems are bounded from below, which we will express by choosing a lower bound of $0 \leq(\psi, H \psi)$ " (2017: 326). This epistemic fact seems to be promoted to a general condition that a time-reversal operator must meet for its acceptability, meaning that $\langle\psi, H \psi\rangle$ and $\langle T \psi, T H \psi\rangle$ must be both non-negatives. Next, Roberts demands that there is at least "one realistic dynamical system" that satisfies timereversal invariance in the sense that satisfies $T e^{i t H} \psi=e^{-i t H} T \psi$. Roberts makes the point that the time-reversal operator is demanded to be anti-unitary in order to meet these requirements, so for reduction, he assumes that such an operator is unitary. This leads to it $H=-i t T H T^{-1}$ and thus to $T \mathbf{H} T^{-1}=-H$. What we finally get is $0 \leq\langle\psi, H \psi\rangle=$ $-\langle T \psi, T H \psi\rangle \leq 0$ and this result leads to either accept that the Hamiltonian is unbounded from below (what he had previously ruled out) or that the Hamiltonian is the operator zero, which renders triviality. Therefore, by reductio, the time-reversal operator cannot be unitary but anti-unitary.

\section{The Philosophical Background of the Orthodoxy}

Let me briefly summarize what I have shown so far. First, I outlined the mathematical tailoring of time reversal in QM according to the orthodoxy. What we learnt from it is that time reversal must be given by an anti-unitary implementation, since it sensibly captures a notion of time reversal that shares some similarities with how time reversal is thought of in Hamiltonian classical mechanics. However, as I claimed, the justification of the mathematical tailoring was rather poor, calling for further explanation. This has been partially carried out in Section 3 by laying the physical foundations of time reversal as backtracking in QM. Regardless how much satisfactory this justification may be, a question remains: what entitles us to conceive of time reversal as backtracking. In other words, what is the philosophical background upholding the association between time reversal and backtracking. In this section I will look into these philosophical aspects.

Such philosophical background is a second step in the conceptual justification of the orthodoxy in QM. What I will argue is that this philosophical background mainly consists of two pillars:

- A relationalist view of time, which motivates a functional reductionist approach to time reversal in terms of motion reversal.

- A by-stipulation view of symmetries, which postulates that fundamental equations of motion must remain invariant under time reversal.

Only upon this philosophical background, the physical justification of Section 3 holds, and with it, the orthodoxy altogether. It is worth stressing that any rejection of either of the pillars would produce some conceptual instability in the orthodoxy, loosing persuasive force. 
Consequently, much of its persuasive force, I claim, relies on accepting the philosophical background. What do I mean by this? The philosophically interesting question is why we decide to call a specific piece of mathematics 'time reversal'. Naturally, the mathematical tailoring does not provide any answer to it. The physical justification just does it partially, since it chiefly concerns the association of time reversal with backtracking in quantum mechanical terms, and whereby, with the anti-unitary implementation. However, nothing has been said so far, on the one hand, why time reversal should be understood as backtracking to in the first place, and on the other, what general principles guide such an understanding. Responses to such questions can be only found within the philosophical background of the orthodoxy.

\subsection{Leibniz meets time reversal at the Plank scale.}

My first thesis is that the notion of time reversal that the anti-unitary operator aims to implement presupposes a relationalist view of time. This, in turn, motivates a functional reduction of time reversal in terms of motion reversal.

Relationalism with respect to time was famously championed by Leibniz. In his third letter to Samuel Clarke (dated February 25, 1716), he claimed that:

"what that argument really proves is that times, considered without the things or events, are nothing at all, and that they consist only in the successive order of things and events"

Our philosophical understanding of time plays a role in our conceptual understanding of time reversal -If we are said to invert the direction of time, it seems at least reasonable to suppose that our course of actions will be different depending on what we understand by 'time'. And in this sense our metaphysics of time comes first: It determines not only what time reversal is but also upon what it is meant to act. In other words, it underpins our conceptual understanding of time reversal, which in turn guides the mathematical tailoring as shown in Section 2.

There are many different types of relationalist-like views in metaphysics and in philosophy of physics that, in general, share the idea that time is nothing over and above temporal relations among events and things (Benovsky 2010: 492), though they can greatly vary on which it is considered as objective and fundamental in the physical world (see Sklar 1974, Earman 1989, Pooley 2013, for comprehensive overviews of the different kinds of relationalisms). To keep things simple, I will consider a relationalist-like view on time as holding two tenets:

R1 A monist ontology. There are only events or physical bodies in the world and their (space) temporal relations.

R2 A reductionist attitude. Time is nothing but change. The sort of relation between the physical world and the concept of 'time' is that of a Leibnizian 
representation or a Machian abstraction: time is an ideal, unreal entity parasitic on events-things' changing.

According to these tenets, the variable $t$ occurring in most physical theories (setting aside general relativity) is merely an external and unreal parameter, which should not be taken as representing anything with physical meaning. The folk wisdom is that a relationalist metaphysics of time implies some reductionist attitude toward temporal predicates. For instance, any reference to the 'directionality of time' should not be taken literally as if there were some primitive entity exemplifying the property of directionality. Rather, it should be taken metaphorically -the 'directionality of time' boils down to the directionality of the change of a series of temporal relations held by their relata.

When I claim that the orthodoxy upholds a relationalist metaphysics of time, I chiefly mean it does not take time reversal literally, as if it were a transformation of time itself (whatever it might mean in physical terms). In fact, the parlance of time reversal in physics and philosophy of physics is mostly metaphorical (see, for instance, Wigner 1932: 325, Gibson and Pollard 1976: 177, Ballentine 1998: 377). The task for the philosophical reflection on time and time reversal is thus to conceptually articulate the underlying notions and elements converging into the idea of time reversal as orthodoxly understood. So, the relationalist metaphysics of time makes coherent a series of assumptions and elements that construe the orthodoxy.

According to the second relationalist tenet, the notion of "time" is just an abstraction (ens rationis) drawn from change, so the ' $t$ ' is not actually representing anything physical, but just an abstract parameter. In consequence, one of the most salient conceptual features of time reversal from a relationalist-like view is that the $T: t \rightarrow-t$ transformation must not be taken too seriously. It would be naïve to take $t \rightarrow-t$ as performing a physically relevant action upon dynamical equations. What is really substantive in the understanding of time reversal is not the transformation of $t$, but the transformation of change. This suggests that time reversal should be considered as a "shortcut" standing for dynamically relevant transformations related to the change (or motion) of a system. To put it in a slogan, a relationalist understanding of time reversal holds that time reversal is nothing but change (or motion) reversal. This is the overarching concept grounding the physical justification and guiding the mathematical tailoring: the formal representation ultimately ought to capture the idea of reversing the change, whatever it comes to mean within a physical theory.

So, we can postulate as a general scheme the following properties of a relationalist view of time reversal

$\boldsymbol{T}_{\text {Rel }} \quad$ (a) A mere re-parametrization of $t$ by $T: t \rightarrow-t$

(b) A change of all dynamically relevant magnitudes so as to generate a backward evolution, which is expressed by extensionally specifying the 
dynamically relevant transformations to take a system back to its original state.

The physically substantive part is given by the property (b), which genuinely generates the symmetry transformation. And it is important to highlight that the two physical justifications I laid out in Section 3 are greatly based philosophically on this property: it motivates why certain observable has to transform in a specific way to generate the relevant symmetry transformation, namely, time reversal as motion reversal.

The second property can be clarified by proposing a sort of functionalist reduction of time reversal to motion reversal. Such an analysis will show how motion reversal realizes time reversal. The literature on functionalism (and, particularly, functional reduction) is abundant, so I will not get into details here. In general, it has mainly focused on either the relation between the mental and the physical in philosophy of mind, or the relations between high-order properties used in special sciences and low-order properties more frequent in physics. What I propose here is not a strict functional reduction as the one discussed in philosophy of mind or general philosophy of science, but a style of reasoning which can quite well capture the metaphysical and epistemic relations holding between time reversal and motion reversal in physics (for a functionalist approach in philosophy of physics, see for instance, Knox 2018).

The overall idea is that motion reversal (and those properties attached to it) realizes time reversal (and thus all those subsidiary properties attached to it). In particular, the notion of time reversal is functionally reduced to the idea of 'backtracking', in the sense that 'time reversal' refers to dynamical realizers that play the role of retracing a system's state to its origin. So, we can rephrase this by saying that if the state of a system has been "timereversed", or that the history of a system has been "time-reversed", we have to find the realizers of such a state and such a history in terms of the dynamical operations that effectively generate a backtracking process. From an abstract perspective, the notion of time reversal is simply a placeholder, whose occupants will be those realizers playing the role of retracing a system's state to its origin. The problem of working out the right form of the timereversal transformation is that of working out the right realizers within a specific physical theory.

The idea could be developed even further by offering the Ramsey sentence of time reversal, which has the structure

$$
\exists X, \exists Y, \exists Z(\ldots X \ldots Y \ldots Z)
$$

What we know is that the notion of backtracking or (change) motion reversal realizes the notion of time reversal. What we have to do now is to supply the roles of each the key players (X, Y and Z) and to identify which specific transformations within a particular physical theory play each role. This realizes Ramsey sentence consequently. Then, in order to apply 
time reversal properly, we have to provide the ordered $n$-tuple of realizers within a physical theory that satisfies the Ramsey sentence for time reversal.

Under this framework, we could either adopt an eliminativist or conservative attitude. The eliminativist will be prone to simply eradicating any temporal predicate and structure in favor of predicates and structures exclusively referring to change. This would automatically remove temporal predicates from the physical picture when we want to be rigorous about what we are really doing when time reversing an equation of motion or a physical system. The term 'time reversal' is just flatus vocis (see Rovelli 2004 for such a radical attitude with respect to time). The conservative reductionist will be rather prone to preserving some temporal predicates and structures, though acknowledging their actual realizers relate to change. This view better preserves the classical Leibnizian-Machian framework, where durations are relative, time is essentially change, but other traditional temporal structures remain absolute (see Gryb and Thébault 2016 for a defense of this more conservative relationalism in quantum gravity).

When we center in the physical justification of the orthodoxy, the relationalist view of time-reversal explains in a natural way why we should understand time reversal as a twotime evolution, basing Wigner's general criterion. If time reversal is functionally realized by motion reversal, then any fair formal implementation of it has to pose the enough structure to represent the reversion of motion. In Wigner's general criterion, this is provided by guaranteeing that the time(-motion)-reversal transformation generates a second time translation that takes the evolution of the state back to its origin. Otherwise, the implementation of time(-motion) reversal fails to genuinely capture the idea of backtracking. But what guarantees the viability of the argumentation is the two relationalist tenets. To be clear, this is not about the mathematical tailoring (as often argued when discarding alternative implementations of time reversal), but about metaphysical commitments. The mathematical tailoring just follows the definition established metaphysically.

The same tenets also ground the Hamilton's spectrum argument I presented in Subsection 3.2. Someone might nonetheless bring up the following argument against. The Hamiltonian is a first-time-derivative magnitude, so it is natural to expect the Hamiltonian to change its sign under time reversal, which would lead to transforming a bounded-from-below Hamiltonian into an unbounded-from-below Hamiltonian. However, from a relationalist understanding of time reversal the demand is excessive. Whether physical magnitudes are canonically defined as first-time derivative does not play any substantive role in defining time reversal, because we are not interested in $t$. We should instead focus on elucidating what role such physical magnitudes play in the evolution of the state and what role they should play if the evolution were reversed. The conceptualization of time reversal at a physical level precisely attempt to work that out. The real issue is not whether or not it makes sense that the Hamiltonian changes its sign under time reversal, but whether such a transformation plays 
any role in formally implementing time reversal as backtracking. Clearly, it does not, but quite the opposite. This explains why the unitary transformation must be discarded

Any defense of the anti-unitary representation of time reversal in QM makes sense because the physical justification of time reversal makes sense. But it is crucial to understand that the physical justification makes sense only if it is at least developed within a relationalist understanding of time, where time reversal is functionally realized by change (motion) reversal. This would entail that if for any reason we reject any of the relationalist tenets, the physical justification of time reversal loses much of its persuasive force, dragging naturally down the anti-unitary representation of time reversal. This does not favor directly a heretic approach to time reversal in QM. Neither do I aim to feed the controversy between alternative implementations of time reversal in QM. What I want to emphasize is the relevance of the metaphysical background for the orthodoxy -It does not merely play a subsidiary role in our physical understanding of time reversal, but it grounds the physical justifications and, consequently, the mathematical tailoring. The orthodoxy is not merely an uncommitted defense of a particular mathematical tailoring, but a well-articulated general view on time reversal. The philosophical background is so important for the orthodoxy that once we take a brick away from the building, the whole structure gets unstable.

\subsection{Time-Reversal Invariance: by-stipulation or by-discovery}

In the previous section, I focused on the metaphysical pillar of the orthodoxy. In this section, I will center in the second pillar, which concerns epistemic and heuristic aspects of time reversal in QM. Whereas the first pillar chiefly centered in the time-reversal transformation (what we metaphysically and physically mean by 'time reversing'), the second pillar rather centers in the status of symmetries in physics. To be precise, it centers in the epistemic and heuristic aspects that connect the construction of a time-reversal transformation to the role that the time-reversal symmetry should play in a physical theory.

There are at least two opposing views on space-time symmetries in modern physics. One of them, which I will call by-stipulation, takes symmetries as postulated, being true independently of the details of the dynamics. The other, which I will call by-discovery, takes symmetries as a result of the details of the dynamics. In the former case, symmetries are principles that constrain the dynamics. In the latter, symmetries are derived from it. As timereversal is prima facie a space-time symmetry, both views are also present in this case. What I will argue is that the orthodoxy presupposes a by-stipulation view of time-reversal invariance, which offers, to a great extent, support to the physical justifications of an antiunitary implementation. To say it differently, the anti-unitary implementation of time reversal is the only formal representation capable of satisfying a by-stipulation view of timereversal invariance in QM.

What does justify the distinction between by-stipulation and by-discovery symmetries? Katherine Brading and Elena Castellani show that space-time symmetries are sometimes 
thought of as guides to theory construction. That is, principles that must be satisfied whatever the final details of the theory come to be. The mechanism whereby a symmetry is raised to a must-satisfied principle is that of stipulation -we postulate, independently of the details of a theory's dynamics, that a given symmetry holds, then the dynamics adapts to the symmetries' constraints. When laying the groundwork for Bohmian Mechanics, Dettlef Dürr an Stephan Teufel for instance write

"A symmetry can be a priori, i.e., the physical law is built in such a way that it respects that particular symmetry by construction. This is exemplified by spacetime symmetries, because spacetime is the theater in which the physical law acts (as long as spacetime is not subject to a law itself, as in general relativity, which we exclude from our considerations here), and must therefore respect the rules of the theater". (2009: 43-44)

It is worth contrasting this quote to others we can find in the literature on symmetries. John Earman says

"The received wisdom about the status of symmetry principles has it that one must confront a choice between the a posteriori approach (a.k.a. the bottom up approach) versus the a priori approach (a.k.a. the top down approach)". (2004: 1230)

Earman's distinction goes along with that of Brading and Castellani's (2007): whereas some take symmetries as postulated, guiding theory construction, others follow an opposite trend, according to which symmetries are a consequence of specific laws like a discovery (2007: 1347). The idea of postulating a symmetry is normative, suggesting certain degree of necessity: a theory's dynamics must satisfy the symmetry principles, even though if the dynamics had been different. This gives symmetry principles certain modal robustness (or counterfactual robustness, see Lange 2009), entailed by its normative nature.

Interestingly, Earman (1989) suggests that symmetry principles should be considered contingent, rather than necessary:

"it would seem that the symmetry transformation could not fail to be a true symmetry of nature, contradicting the usual understanding that symmetry principles are contingent, that is, are true (or false) without being necessarily true (or false)" (1989: 121)

Earman's quote rejects the normative nature of symmetry principles, which, implicitly or explicitly, leads to an opposing view wherein space-time symmetries as a consequence of the details of a theory's dynamics -it happens that a given dynamics has some space-time symmetries and lacks others, and this is we come to know by investigating the formal relations held by the elements in differential equations. Remarkably, this approach was following by Isaac Newton in formulating classical mechanics in the Principia as the 
relativity principle appears as a corollary of the equation of motion (Corollary 5, see also Brading and Castellani 2003: 6) In this sense, a space-time symmetry plays a descriptive role, rather than a normative.

To write all this out neatly, both approaches can be defined as follows. For a general space-time symmetry $\sigma$ :

By-stipulation approach $\sigma$-symmetry plays a normative role in a theory's dynamics and it must thereby be regarded as a priori and necessary for a theory $\mathrm{T}$.

By-discovery approach $\sigma$-symmetry plays a descriptive role and it must thereby be regarded as a posteriori and contingent for a theory $T$.

It is worth remarking that the epistemic notions of "a priori/a posteriori" should be understood not in the traditional sense (independent or not of the experience), but in relation to a theory's dynamics: whether $\sigma$-symmetry is known independently of a theory's laws.

Time-reversal invariance can be regarded from both approaches. My claim is that the orthodoxy presupposes a by-stipulation view of time-reversal invariance since it plays a normative role in the theory construction as well as in the mathematical tailoring of the timereversal transformation. To illustrate the first point, Robert Sachs says:

"In order to express explicitly the independence between the kinematics and the nature of the forces, we require that the transformations leave the equations of motion invariant when all forces or interactions vanish" (Sachs 1987: 7)

Time-reversal symmetry is required to hold by stipulation in the case of the free Schrödinger equation, that is, in the evolution of free-interaction quantum systems. This idea nicely comes along with the "theater picture" of Dürr and Teufel: the simplest systems' dynamics reflect genuinely the structure of the theater, both its asymmetries and symmetries. But such a structure is pre-existent and independent of the dynamics' systems, playing the role of setting the (space)-time background for all models of the theory and of individuating the nature of forces, interactions and the various structures (i.e. asymmetries) they generate.

To illustrate the second point, let us briefly move to a different theory -classical electromagnetism. In discussing the mathematical form of the time-reversal transformation, Frank Arntzenius and Hillary Greaves (2009) claim that a widespread account, which they call 'the textbook account', proceeds as follows (see also Peterson 2015):

"Next let us consider the electric and magnetic fields. How do they transform under time reversal? Well, the standard procedure is simply to assume that classical electromagnetism is invariant under time reversal. From this assumption of time reversal invariance of the theory (...) it is inferred that the 
electric field $\mathrm{E}$ is invariant under time reversal (...)" (Arntzenius and Greaves 2009: 6. Italics mine)

The same mechanisms, mutatis mutandis, guides the anti-unitary implementation of time reversal in QM. In more explicit terms, the stipulation of time-reversal invariance justifies to a great extent why the implementation of time reversal in QM must be anti-unitary. This is more evident when we contrast with the unitary implementation: If we previously presume that time-reversal invariance holds, then the formal implementation of time reversal cannot be one that make the free Schrödinger equation (eq. 2.9) non-time-reversal invariant. Such a result would be in fact at odds with Sachs' quote too: we, for instance, stipulate that the free Schrödinger equation is time reversal invariant in order to express the independence between dynamics and kinematics. Hence, its stipulation plays a heuristic role in our understanding of the theory, which we will be missed if the unitary transformation is rather adopted. So, everything ends up at the same place: the anti-unitary operator is the only implementation that carries out the sort of required transformations to keep the free Schrödinger equation invariant, satisfying the epistemic and heuristic stipulation.

This by-stipulation view of symmetries and the implied justificatory mechanism can be also regarded from a different angle. The stipulation of time-reversal invariance appears as a premise in the characterization of the time-reversal operator, guiding the mathematical tailoring. Recall Wigner's definition of time reversal in Section 3. He invokes two explicit premises:

1. that a suitable time-reversal transformation must be able to restore "the system to its original state" (1932: 326),

2. and that time inversion must flip the direction of momentum to compensate for the twofold application of $T$ in Wigner's general criterion.

But there is also one fundamental implicit assumption in requiring time reversal to meet Wigner's general criterion:

3. for a time-reversal transformation to be well-defined (and to exist at all), the second time translation (from $t_{2}$ to $t_{1}$ ) must also be physically possible.

To see how this last assumption works let's suppose that a quantum state $|\psi\rangle$ evolves from $t_{1}$ to $t_{2}$, according to the Schrödinger equation (first time translation). At $t_{2}$, time reversal is applied upon the Schrödinger equation. If the time-reversal transformation is well-defined, then the time-reversed state $|\psi\rangle^{T}$ should evolve from $t_{2}$ to $t_{1}$ also conforming with the Schrödinger equation (second time translation). And here the implicit assumption comes in. According to Wigner, the operation to be applied upon the state at $t_{2}$ must be of such a kind that yields a quantum-mechanical evolution -the transformation takes a solution of the free 
Schrödinger equation and transforms it into a solution of the free Schrödinger equation This is the standard definition of symmetry and the state that satisfies is $|\psi\rangle^{T}=\left|\psi^{*}\right\rangle$.

To complete the argument, let us now suppose that at $t_{2}$, we apply a unitary time-reversal operator. As remarked above, the free Schrödinger equation will not temporally translate the system back. But, even worse, the transformation will fail to generate the second time translation, turning a solution of the free Schrödinger equation into a non-solution. The metaphysical pillar of the orthodoxy shows that such a transformation is ill-conceived because it fails to represent time reversal as motion reversal. The by-stipulation symmetry view shows that such a transformation is ill-conceived because it makes the free Schrödinger equation non-time-reversal invariant. As I commented before, whether we assume a bystipulation or a by discovery view is motivated by various reasons, mainly concerning epistemic and heuristic features. Which view to take is not at issue here. What it is at issue is the justificatory role that the by-stipulation view plays when it comes to the mathematical tailoring of time reversal in QM -the anti-unitary representation is the only implementation of time reversal that satisfies such a view along with the potential theoretical virtues attached to it.

\section{Concluding remarks}

In this paper I have offered novel insights to address the debate on time reversal in QM. I began by distinguishing three steps in construing the orthodoxy: the mathematical tailoring (Section 2), the physical justification (Section 3), and the philosophical background (Section 4). Each step was shown to be supported by an underlying one: the mathematical tailoring depends on its physical justification, which only makes sense within a philosophical background. The general aim was to bring to light the relevance of the philosophical background as a series of philosophical commitments upon which the orthodoxy hinges. With respect to this, I have claimed that the orthodoxy is philosophically supported by two pillars:

- a relationalist metaphysics of time, which promotes a functionalist reduction of time reversal in terms of motion reversal

- a by-stipulation view of time-reversal invariance

What this primarily shows is that the orthodoxy is not philosophically neutral, but it substantially relies on a series of metaphysical, epistemic and heuristic commitments. These play a major justificatory role in the anti-unitary implementation of time reversal in QM. Contrarily to how the debate has been developed thus far, the quid of the notion of time reversal in QM should not be primarily framed in terms of whether it ought to be anti-unitary or unitary, but if we have well-grounded reasons to uphold such two pillars. Any discussion on time reversal in QM, I deliver, should start from them. 
So, what grounds the claim that a quantum system has been genuinely time reversed is a well-articulated view that is not simple, but quite complex, involving various mathematical, physical and, fundamentally, philosophical assumptions. The latter directly relate to big questions such as the nature of time and symmetries in physics in metaphysics, around which philosophers and scientist have long been gravitating. And it is such philosophical complexity what feeds the orthodoxy's persuasive force, rather than any seemingly obviousness or self-evident truth.

\section{References}

Abraham, R. and Marsden, J. E. (1978). Foundations of Mechanics, 2nd edn, Addison- Wesley Publishing Company, Inc.

Albert, D. Z. (2000). Time and Chance. Cambridge, MA: Harvard University Press.

Arntzenius, F. and Greaves, H. (2009). "Time reversal in classical electromagnetism". The British Journal for the Philosophy of Science, 60: 557-584

Ballentine, L. (1998). Quantum Mechanics. A modern Development. Singapur: World Scientific.

Barbour, J., and Bertotti, B. (1982). Mach's principle and the structure of dynamical theories. Proceedings of the Royal Society A, 382, 295-306.

Benovsky, J. (2010). "The relationalist and substantivalist theories of time: foes or friends?" European Journal of Philosophy, 19 (4): 491-506.

Brading, K. and E. Castellani. (2007). "Symmetries and Invariances in Classical Physics". Handbook of the Philosophy of Science, Philosophy of Physics, Part B. Eds. J. Butterfield and J. Earman. The Netherlands: Elsevier, 1331-1367.

Callender, C. (2000). "Is time 'handed' in a quantum world?" Proceedings of the Aristotelian Society, 100: 247-269.

Chevallier, G. (2007). "Wigner's theorem and its generalizations". In K. Engesser, D. M. Gabbay and D. Lehmann (eds.), Handbook of Quantum Logic and Quantum Structures, New York: Elsevier Publisher, pp. 429-475.

Costa de Beauregard, O. (1980). "CPT invariance and interpretation of quantum mechanics", Foundations of Physics, 10: 513-530.

Dürr, D. and Teufel, S. (2009). Bohmian Mechanics: The Physics and Mathematics of Quantum Theory, Berlin: Springer-Verlag.

Earman, J. (1989). World enough and space-time. Absolute versus relational theories of space-time. Cambridge, Massachusetts: MIT Press.

Earman, J. (2002). "What time-reversal invariance is and why it matters". International Studies in the Philosophy of Science, 16: 245-264.

Earman, J. (1989). World enough and space-time. Absolute versus relational theories of space-time. Cambridge, Massachusetts: MIT Press.

Gasiororowicz, S. (1966). Elementary Particle Physics. New York: John Wiley and Sons.

Gibson, W.M. and Pollard, B.R. (1976). Symmetry Principles in elementary particle physics. Cambrige: Cambridge University Press. 
Gryb, S. and Thébault, K. (2016). “Time remains”. British Journal for Philosophy of Science, 67: 663-705.

Knox, E. (2018). "Physical relativity from a functionalist perspective". Studies in History and Philosophy of Modern Physics, 67: 118-124.

Lopez, C. (2019). "Roads to the past: how to go and not to go backward in time in quantum theories". European Journal for Philosophy of Science, 9: 27.

Messiah, A. (1966). Quantum Mechanics. New York: John Wiley and Sons.

Peterson, D. (2015). "Prospect for a new account of time reversal". Studies in History and Philosophy of Modern Physics, 49: 42-56.

Pooley, O. (2013). Substantivalist and relationalist approaches to spacetime, In Batterman, R. (Ed.) The oxford handbook of philosophy of physics (pp. 522-586). Oxford: Oxford University Press.

Roberts, B. (2017). “Three myths about time reversal invariance”. Philosophy of Science, 84, 2: 315334.

Roberts, B. (2018). “Time reversal” http://philsci-archive.pitt.edu/15033/1/Roberts2018-Time Reversal.pdf.

Rovelli, C. (2004). Quantum gravity. Cambridge: Cambridge University Press.

Sachs, R. (1987). The Physics of Time Reversal. London: University Chicago Press.

Sklar, L. (1974). Space, Time and Spacetime. Berkeley: University of California Press.

Uhlhorn, U. (1961). "Representation of Symmetry Transformations in Quantum Mechanics.” Arkiv för Fysik 23:307-40.

Wigner, E. (1932). Group Theory and its Application to the Quantum Mechanics of Atomic Spectra. New York: Academic Press (1959). 